\title{
Paysages et otium au début du Haut-Empire
}

\author{
Anne Gangloff
}

Là, tout n'est qu'ordre et beauté, luxe, calme et volupté

BAUDELAIRE, L'invitation au voyage

Le phénomène culturel des villas de luxe, qui est né de l'afflux des ressources apportées par la conquête de la Grèce et du bassin oriental de la Méditerranée, et qui connaît son épanouissement de la première moitié du I ${ }^{\text {er }}$ siècle av. J.-C. à la fin du $\mathrm{II}^{\mathrm{e}}$ siècle ap. J.-C., surtout le long du littoral près de Rome, autour de la baie de Naples et en Toscane, est si étroitement associé à l'idéal aristocratique de l'otium que ces villas sont couramment qualifiées par les archéologues de «villas à otium». Cette notion d'otium n'est cependant pas univoque : elle ne se fixe qu'au I $^{\mathrm{er}}$ siècle av. J.-C., pour désigner la tranquillité de l'existence privée, rendue possible par un détachement temporaire des activités politiques et tournée vers les activités culturelles 1 . Cicéron est le premier à faire apparaître, de manière explicite, ce que J. Bodel appelle la «Villaculture », en ayant fait des villas des aristocrates de l'époque tardo-républicaine le cadre de ses dialogues littéraires ${ }^{2}$.

1 Je remercie, pour leurs relectures et remarques, J. Alerini, M. Fuchs, G. Gorre, N. Haechler, M. Horster, S. Lefebvre et J. Trinquier, ainsi que, pour leurs suggestions, R. Auvertin, F. Carlà-Uhink et S. Wyler. Sur la notion d'otium, voir l'ouvrage classique de J.-M. André, L'Otium dans la vie morale et intellectuelle romaine, des origines à l'époque augustéenne (Paris 1966). Les principales éditions et traductions utilisées sont les suivantes: Pline le Jeune, Lettres. Tome I, Livres I-III, texte établi, traduit et commenté par H. Zehnacker (Paris 2009); tome II, Livres IV-VI, texte établi et commenté par H. Zehnacker, traduit par N. Méthy (Paris 2011); tome III, Livres VII-IX, texte établi et commenté par H. Zehnacker, traduit par N. Méthy (Paris 2012). Sénèque, Lettres à Lucilius. Tome III, Livres VIII-XIII, texte établi par F. Préchac, traduit par H. Noblot (Paris 1957).

2 J. Bodel, < Villaculture >, dans J.A. Becker et N. Terrenato (éds.), Roman Republican Villas. Architecture, Context, and Ideology (Ann Arbor 2012), 45-6o, part. 53-54. 
L'objectif de cette contribution est d'étudier les rapports entre la notion d'otium, elle-même fondamentalement liée à la contemplation ${ }^{3}$, et les paysages intégrés dans la villa - paysages naturels ou paysages peints. Nous employons le terme de paysage dans un sens proche de sa conception courante, en le considérant comme une «étendue de pays » (qui est le sens premier du mot quand il apparaît en 1549) offerte à la vue d'un spectateur ${ }^{4}$, ce qui permet d'englober aussi bien le paysage naturel - plus ou moins marqué par l'intervention humaine - que le paysage artialisé des tableaux. Il s'agit d'examiner, à partir d'exemples précis, l'évolution des interactions entre otium intellectuel, appréhension du paysage et architecture de la uilla urbana au début du Haut-Empire.

On peut partir de la lettre 86 dans laquelle Sénèque décrit sa visite de la villa de Scipion l'Africain à Liternum en Campanie, villa qu'il évoque aussi dans la lettre 51. C'était une villa bien connue, mentionnée aussi dans d'autres sources et devenue à l'époque de Sénèque un lieu de tourisme en raison de la gloire attachée à son propriétaire qui était un exemplum ${ }^{5}$. Il s'agit aussi d'une des premières uillae urbanae que l'on connaisse - mais pas archéologiquement -, qui fut construite au tout début du $\mathrm{II}^{\mathrm{e}}$ siècle av. J.-C.

J'ai vu cette villa toute en pierres de taille, le mur enfermant la forêt, les tours de défense des deux côtés de l'entrée; en contrebas des constructions et des massifs de verdure, masquant une citerne qui suffirait aux besoins d'une armée entière, le cabinet de bain étroit, ténébreux, selon la coutume du vieux temps. Il fallait l'obscurité à nos ancêtres pour qu'ils se sentissent bien au chaud. Je pris un très vif plaisir à considérer la manière de vivre de Scipion par rapport aux habitudes d'aujourd'hui. Dans ce réduit la terreur de Carthage, le héros à qui Rome doit de n'avoir été qu'une fois prise, baignait son corps fatigué de rustiques travaux ; car il peinait aux champs et, comme un citoyen des âges antiques, conduisait lui-même la charrue. Le grand homme a tenu sous ce plafond sordide;

3 Sen. dial. 8 (De otio), 5.3-8.

4 Voir les définitions courantes tirées du dictionnaire Robert que proposent A. Rouveret, «Pictos ediscere mundos : perception et imaginaire du paysage dans la peinture hellénistique et romaine », Ktèma 29 (2004), 325-344, part. 342 (à partir de la description que fait Pline le Jeune de la vue panoramique depuis sa villa de Toscane, que nous verrons plus loin), et J.-M. Croisille, Paysages dans la peinture romaine. Aux origines d'un genre pictural (Paris 2010), 12.

5 Liv. 38.52.1; Sen. epist. 11.86 ;Val. Max. 2.10.2 ; l'otium de Scipion l'Africain est aussi mentionné, également à titre d'exemple, par Cic. off. 3.2.1. Voir J. Henderson, Morals and Villa's in Seneca's Letters : Places to Dwell (Cambridge, 2004), part. 93-117, 158-170. 
voici le vil pavé qu'ont foulé ses pas! À présent, qui souffrirait de se baigner dans de semblables conditions? On se regarde comme pauvre et crasseux, si les parois de la salle de bain ne diffusent l'éclat de grands miroirs ronds, de prix, si les marbres humides ne s'incrustent, pour un effet de contraste, dans ceux d'Alexandrie, si l'on ne voit régner autour de ces plaques de marbre un filet d'émail dont la mosaïque savante rappelle le pinceau du peintre [...]. Dans ce bain de Scipion il y a, au lieu de fenêtres, des meurtrières taillées en plein mur pour laisser entrer la lumière sans nuire à la défense du castel. Aujourd'hui on appelle trous à mites les bains qui ne sont pas agencés de façon à recevoir par d'immenses fenêtres le soleil toute la journée, qui ne permettent pas de se tremper et de se hâler la peau dans le même temps, où l'on n'a pas vue, de sa baignoire, sur la campagne et la pleine mer6.

Sénèque présente donc la villa de Scipion l'Africain comme une sorte de ferme fortifiée, dont il souligne la modestie. Ce qui est intéressant est qu'on a pu montrer, tout au contraire, que cette villa fut luxueuse en son temps, d'une part parce que son plan paraît correspondre au type de plan tetrapyrgos, qui est un plan rectangulaire fermé, caractérisé par la présence de quatre tours angulaires et d'espaces organisés autour d'une cour centrale entourée d'un péristyle. Ce type, rare dans le paysage romain, était celui d'un palais hellénistique (par exemple celui de Démétrios Poliorcète à Démétriade), ce qui en faisait un cadre bien approprié à un imperator romain 7 . Le caractère luxueux est impliqué, d'autre part, par la présence d'un balneum, alors que celui-ci n'a

6 Sen. epist. 11.86.1-9: Vidi uillam extructam lapide quadrato, murum circumdatum siluae, turres quoque in propugnaculum uillae utrimque subrectas, cisternam aedificiis ac uiridibus subditam quae sufficere in usum uel exercitus posset, balneolum angustum, tenebricosum ex consuetudine antiqua: non uidebatur maioribus nostris caldum nisi obscurum. Magna ergo me uoluptas subiit contemplantem mores Scipionis ac nostros: in hoc angulo ille 'Carthaginis horror', cui Roma debet quod tantum semel capta est, abluebat corpus laboribus rusticis fessum. Exercebat enim opere se terramque (ut mos fuit priscis) ipse subigebat. Sub hoc ille tecto tam sordido stetit, hoc illum pauimentum tam uile sustinuit: at nunc quis est qui sic lauari sustineat? Pauper sibi uidetur ac sordidus nisi parietes magnis et pretiosis orbibus refulserunt, nisi Alexandrina marmora Numidicis crustis distincta sunt, nisi illis undique operosa et in picturae modum uariata circumlitio praetexitur [...]. In hoc balneo Scipionis minimae sunt rimae magis quam fenestrae muro lapideo exsectae, ut sine iniuria munimenti lumen admitterent; at nunc blattaria uocant balnea, si qua non ita aptata sunt ut totius diei solem fenestris amplissimis recipiant, nisi et lauantur simul et colorantur, nisi ex solio agros ac maria prospiciunt.

7 L. Romizzi, Villa d'Otium dell'Italia antica (II sec. a. C.-I sec. d. C.) (Naples 2001), 120-121, cat. 64 pour l'exemple concret de la villa de Mazara del Vallo en Sicile ( $\mathrm{II}^{\mathrm{e}}$ siècle av. J.-C.). 
été couramment intégré à l'architecture de la villa qu'au début de l'époque impériale ${ }^{8}$.

La description de Sénèque est idéologique, comme le montre l'association qu'il fait, par exemple, entre Scipion l'Africain et Caton l'Ancien, autre propriétaire d'une fameuse uilla rustica ${ }^{9}$. On peut faire le parallèle avec Varron qui, dans les Res rusticae, a opposé uilla urbana et uilla rustica d'une manière artificielle - qui ne correspondait pas à une réalité beaucoup plus complexe pour mettre en avant les traditions romaines dans le contexte troublé de la fin de la République ${ }^{10}$. Sénèque aussi fait retour à un idéal traditionnel du soldat-paysan, par opposition au luxe des villas à otium et des jardins, dont le premier promoteur - certes atypique - fut naturellement, à son époque, l'empereur Néron. En faisant construire la Domus Aurea, celui-ci introduisit à Rome même la plus luxueuse des villas à otium ${ }^{11}$. Sénèque a écrit les Lettres à Lucilius un peu avant la construction de la Domus Aurea, à partir de 63, dans la semi-retraite où il s'est tenu dès la deuxième moitié de l'année 62, contre le gré de l'empereur d'après Tacite.

Dans sa description de la villa de Scipion, le philosophe ne s'attache pas au paysage : il se contente d'évoquer plus loin, rapidement, des éléments du paysage agricole puisqu'il ne s'intéresse qu'à la pars rustica de la villa et à sa fonction de lieu de production agricole. Il souligne l'aspect fermé de l'architecture,

8 Romizzi 2001, op. cit. (n. 7), 39; X. Lafon, « Les bains privés dans l'Italie romaine au II ${ }^{\mathbf{e}}$ siècle av. J.-C. », dans Les thermes romains: actes de la table ronde organisée par l'École française de Rome: Rome, 11-12 novembre 1988 (Rome 1991), 97-114, part. 99-100, 102, 112 ; $\mathrm{X}$. Lafon, Villa maritima. Recherches sur les villas littorales de l'Italie romaine (Rome 2001), 43-47 sur la villa de Scipion.

9 Sen. epist. 5·51.11-12 : sont aussi mentionnés les grands imperatores tardo-républicains, Marius, Pompée et César, propriétaires de villas à caractère «militaire».

10 Varro rust. 1.13.6-7, déplore également le luxe et les extravagances des villas contemporaines, par opposition aux villas du passé, construites pour exploiter les campagnes. Mais à côté de cette opposition idéologique, il établit aussi, à l'intérieur même de la villa, une distinction fonctionnelle entre pars urbana et pars rustica, entre partie résidentielle d'une part, partie productive et partie de stockage de l'autre. Voir A.F. Wallace-Hadrill, «The villa as a cultural symbol», dans A. Frazer (éd.), The Roman Villa: Villa Urbana (Philadelphia 1998), 43-53; M. Zarmakoupi, Designing for Luxury on the Bay of Naples. Villas and Landscapes (100 BCE-79 CE) (Oxford 2014), 5. Sur la persistance de la pars rustica dans la uilla urbana et sur le caractère non fondé de l'opposition entre uilla rustica et uilla urbana, voir J. D'Arms, Romans on the Bay of Naples (Cambridge [MA] 1970), 351-383. Voir aussi N. Purcell, 〈 The Roman uilla and the landscape of production 〉, dans T.J. Cornell et K. Lomas (éds.), Urban Society in Roman Italy (London 1995), 151-179.

11 Suet. Nero 31; Tac. ann. 15.42. Voir N. Purcell, < Town in country and country in town >, dans E.B. MacDougall (éd.), Ancient Roman Villa Gardens (Washington 1987), 187-203; sur la critique par l'élite romaine des maisons de luxe: C. Edwards, The Politics of Immorality in Ancient Rome (Cambridge 1993), 137-172. 
le caractère sombre de la pièce qu'il décrit, le balneum, mettant en évidence, a contrario, l'importance de la vue sur la campagne et la mer au sein de la villa à otium. Ces éléments peuvent être mis en rapport avec d'autres remarques formulées par le philosophe quand il évoque des villas à otium : les voluptés du paysage de Baïes sont perturbantes et nuisent à l'otium du sage ${ }^{12}$; il reconnaît l'influence du pays, de l'environnement sur l'esprit ${ }^{13}$, ce qui va à l'encontre de l'indépendance du sage, qui doit pouvoir, par l'esprit, aller librement d'un lieu à un autre ${ }^{14}$. Le discours de demande de congé à l'empereur que Tacite a attribué à Sénèque est cohérent avec la position exprimée par le philosophe dans ses lettres ${ }^{15}$. Sénèque considère en effet dans ce discours ses propriétés suburbaines et les jardins qu'il a fait aménager comme des possessions inhérentes à son rang social et à sa proximité avec Néron : ils font partie des bienfaits (beneficia) de l'empereur. Le soin consacré aux jardins et aux villas n'est donc pas envisagé comme un élément de l'otium, mais, au contraire, comme un sujet de préoccupation pour l'esprit. L'otium du sage se vit dans la solitude et le repli sur soi ${ }^{16}$, il n'est pas compatible avec le cadre de la villa à otium.

À la fin du $\mathrm{I}^{\mathrm{er}}$ siècle ou dans les premières années du $\mathrm{II}^{\mathrm{e}}$ (probablement entre 97 et 107/108), un autre riche sénateur proche du pouvoir impérial - mais dans un contexte de rapprochement entre celui-ci et l'élite sociale et intellectuelle-, Pline le Jeune, fait la description dans ses lettres de deux de ses villas à otium, celle des Laurentes et celle de Toscane. La villa toscane a été localisée sur le site de San Giustino, dans la haute Vallée du Tibre, à la limite nord de l'Ombrie, à la frontière de la Toscane et des Marches, mais il n'est pas possible à l'heure

Sen. epist. 5·51.11. Sur Baïes comme symbole et comme lieu de villégiature privilégié, notamment par les Julio-Claudiens, voir Lafon 2001, op. cit. (n. 8), 187-204, 243-246 et 257-259.

13 Sen. epist. 5.51.11 (dans la lettre, mer et ville sont opposées à deux éléments positifs de l'environnement que sont la montagne et la campagne); 11.86.14.

14 Sen. epist. 5.55.8.

15 Tac. ann. 14.53-54, part. 53.5: Ubi est animus ille modicis contentus? tales hortos exstruit et per haec suburbana incedit et tantis agrorum spatiis, tam lato faenore exuberat? una defensio occurrit, quod muneribus tuis obniti non debui, «Où est cette âme satisfaite de peu? Est-ce elle qui aménage de tels jardins, qui se pavane dans ces villas suburbaines, qui regorge de domaines aussi vastes, d'un revenu aussi étendu? »; Tac., ann. 54.3: Nec me in paupertatem ipse detrudam, sed traditis quorum fulgore praestringor, quod temporis hortorum aut uillarum curae seponitur, in animum reuocabo, «Sans me réduire moi-même à la pauvreté, j'abandonnerai des biens dont l'éclat m'éblouit, et le temps consacré au soin des jardins ou des villas, je le rendrai à mon esprit » (trad. par P. Wuilleumier). Sénèque semble répondre aussi aux attaques de ses ennemis, qui sous-entendaient dans le passage précédent qu'il allait bientôt surpasser l'empereur par le luxe de ses villas et de ses jardins : Tac. Ann. 14.52.2.

16 Sen. Dial. 8 (De Otio), $1.1 ; 2.1$. 
actuelle de retracer le plan complet de la résidence du propriétaire ${ }^{17}$. Divers sites ont été proposés pour la villa des Laurentes : la villa Plinio ou Palombara à Castel Fusano, au sud-ouest de Rome dans l'Agro Romano ; la villa Grotte di Piastra ou Villa Magna à Castel Porziano, dans la même zone mais un peu plus à l'est que la précédente. Au contraire de ce qu'on a vu pour Sénèque, les descriptions de Pline font émerger une notion de l'otium qui concilie à la fois le luxe architectural de la uilla urbana et des jardins et l'étude intellectuelle ${ }^{18}$.

Les descriptions de Pline relèvent de l'exercice littéraire ${ }^{19}$ : elles s'apparentent à des visites guidées de la uilla urbana, dont la partie rustica, bien développée au moins dans la villa de Toscane puisqu'on sait par sa correspondance que Pline en tirait des revenus ${ }^{20}$, est gommée, sauf comme élément de la beauté du paysage ${ }^{21}$. Il s'agit donc de descriptions codifiées, révélatrices de valeurs et de goûts communs à l'élite aristocratique de son temps, à laquelle Pline s'adresse : ainsi L. Domitius Apollinaris, auquel était destinée la description de la villa de Toscane dans la lettre 5.6, qui fut consul suffect en 97 et fut peut-être le patron de Martial, et Cn. Pedanius Fuscus Salinator, le protégé de Pline, auquel est expliquée l'organisation de l'otium littéraire dans la villa de Toscane, dans la lettre 9.36; Fuscus était le neveu d'Hadrien et il fut consul en 118 avec l'empereur ${ }^{22}$.

Or, ces descriptions de villas sont caractérisées par l'importance accordée au paysage, sur lequel est ouverte l'habitation. L'interaction entre architecture des villas à otium et paysage naturel a été soulignée par M. Zarmakoupi

17 Villa de Toscane: $C I L$ XI 8113, 16 (tuiles trouvées à Tifernum Tiberinum, avec l'inscription C. P[linii] C [aecilii] S[ecundi $]$ ). Voir P. Braconi et J. Uroz Sáez, La villa di Plinio il Giovane a San Giustino : Primi risultati di una ricerca in corso (Pérouse 1999); P. Braconi, « La villa di Plinio il Giovane a San Giustino », dans F. Coarelli et H. Patterson (éds.) Mercator placidissimus. The Tiber Valley in Antiquity. New Research in the upper and middle River Valley (Atti del Convegno, Roma, British School at Rome, 27-28 febbraio 2004) (Rome 2008), 93-108: jusqu'à présent les archéologues n'ont pu fouiller qu'une partie qui ne correspond pas à la résidence de Pline, telle que celui-ci l'a décrite dans sa lettre 5.6.

18 Comme l'a souligné Edwards 1993, op. cit. (n. 11), 142, 163, à la fin du I ${ }^{\text {er }}$ siècle, pour la première fois, on trouve des textes littéraires poétiques (chez Stace et Martial), qui louent le luxe des villas et ne le condamnent plus. Désormais, celui-ci semble «faire partie du paysage ».

19 Plin. Epist. 5.6.40-44 ; A.-M. Guillemin, «Les descriptions de villas de Pline le Jeune», $B A G B 19$ (1928), 6-15, qui renvoie aussi à Stat. Silv. 2.2.

$20 \quad$ Plin. Epist. 4.6 ; 9.36.6, où Pline écarte justement les doléances de ses fermiers comme étant incompatibles avec son otium.

21 Voir sur ce sujet Purcell 1995, op. cit. (n. 10).

22 Pour le premier, voir Mart. 4.86; 7.26 ; 11.15; $P^{2} R^{2}$ D 133. Cn. Pedanius Fuscus Salinator: $P I R^{2} \mathrm{P}$ 20o. L'identification du Gallus auquel était adressée la description de la villa des Laurentes (Plin. Epist. 2.17) est incertaine: $P I R^{2} \mathrm{G}_{59}$. 
dans son livre Designing for Luxury on the Bay of Naples. Villas and Landscapes (10o $B C E-79 C E$ ), à partir d'une étude de cinq villas campaniennes datées entre la fin du $\mathrm{II}^{\mathrm{e}}$ siècle av. J.-C. et la fin du I ${ }^{\mathrm{er}}$ siècle ap. J.-C. ${ }^{23}$. Lauteure a mis en avant, pour expliquer ce phénomène nouveau, propre aux luxueuses villas romaines, aussi bien des innovations techniques comme l'élargissement de la taille des structures en terrasses et la réorganisation du portique et du jardin, qui permettait d'intégrer des paysages naturels au sein-même des villas, que l'apparition d'un goût nouveau pour le paysage, visible notamment dans la poésie bucolique, dès Virgile ${ }^{24}$. Cette ouverture de la villa sur le paysage est manifeste dans les descriptions des villas des Laurentes ou de Toscane, dans lesquelles Pline souligne de manière répétée les vues offertes par les différentes pièces ou structures ${ }^{25}$.

Le goût pour le paysage naturel est également évident, surtout dans la description de la villa de Toscane, où le paysage environnant est décrit de manière continue, comme un panorama ayant sa propre unité, et non comme une succession de topia, comme c'était le cas dans les célèbres définitions du paysage données par Vitruve et Pline l'Ancien ${ }^{26}$ - c'est d'ailleurs cette description qui a contribué à la localisation de la villa de Pline à San Giustino ${ }^{27}$ :

Le pays est d'une grande beauté. Figure-toi un amphithéâtre immense, et tel que la nature seule peut en créer. Une plaine vaste et étendue est entourée de montagnes. Les montagnes, dans leur partie supérieure, portent de hautes et antiques futaies, le gibier y est abondant et varié. Ensuite, des taillis descendent suivant la pente de la montagne. Au milieu, de gras coteaux de terre riche (il n'est pas facile de trouver la roche en aucun endroit quand bien même la chercherait-on) ne le cèdent pas en

23 Zarmakoupi 2014, op.cit.(n.10), sur la villa des Papyri, la villa Oplontis Aà Torre Annunziata, les villas Arianna A et B et San Marco à Stabies. Voir aussi, dans une perspective centrée sur la période tardive, É. Morvillez, « 'Avec vue sur jardin': vivre entre nature et paysage dans l'architecture domestique, de Cicéron à Sidoine Apollinaire», Cahiers «Mondes anciens» 9 (2017), mis en ligne le 16 mars 2017, consulté le 29 mai 2019. URL: http:// journals.openedition.org/mondesanciens/1926 ; DOI: 10.400o/mondesanciens.1926.

24 Zarmakoupi 2014, op. cit. (n. 10), 19-20, 114-122 et 122-139. Sur les liens entre paysages peints et poésie, voir W.E. Leach, The Rhetoric of Space: Literary and Artistic Representations of Landscape in Republican and Augustan Rome (Princeton 1988).

25 Plin. Epist. 2.17.5, 6, 11, 12, 13-15, 20-21 ; 5.6.14, 18, 23, 28, 30. Voir aussi Stat. Silv. 2.2, v. 73-81. L'idéal littéraire de l'ouverture sur l'extérieur est sans doute à relativiser, voir sur ce point la réflexion de Lafon 2001, op. cit. (n. 8), sur les « rapports complexes [que les villas maritimes entretenaient] avec l'extérieur : monde clos ou espace ouvert?», 295-300.

26 Plin. Nat. 35.116; Vitr. 7.5.2.

27 Braconi 2008, op. cit. (n. 17), 93 . 
fertilité aux champs des terrains les plus plats et portent une abondante moisson, qui parvient à maturité seulement plus tardivement, mais non moins bien. À leur pied, sur tous les versants, s'étendent des vignobles, dont le maillage crée, sur un vaste espace, un paysage uniforme. À leur limite, leur bordure inférieure, pour ainsi dire, apparaissent des bosquets. Puis des prairies et des champs, des champs que seuls peuvent entamer d'énormes bœufs et de très puissantes charrues. Si grosses sont les mottes qui se dressent sur le sol très compact, lorsqu'on l'ouvre pour la première fois, que seul en vient à bout le neuvième labour. Les prairies, remplies de fleurs à l'éclat de pierres précieuses, produisent du trèfle et d'autres herbes toujours tendres et souples comme si elles étaient fraîchement écloses; toutes, en effet, sont alimentées par des ruisseaux intarissables. Pourtant, aux endroits où l'eau est la plus abondante, aucun marécage, parce que la terre en pente déverse dans le Tibre tout le liquide qu'elle a reçu sans l'absorber. Le fleuve, propre à la navigation, passe au milieu des champs et transporte à la ville toutes les productions de la campagne, mais seulement en hiver et au printemps. En été, il baisse et le dessèchement de son lit lui fait perdre son nom de fleuve immense ; il le retrouve en automne. Tu auras grand plaisir à contempler le panorama du site du haut de la montagne. Car ce ne sont pas des terres mais un dessin d'une rare beauté que tu auras l'impression de voir. Cette diversité, cette harmonie reposeront ton regard où il se posera. La maison, située au bas d'un coteau, a la même vue que si elle était au sommet ${ }^{28}$.

Plin., epist. 5.6.7-14: Regionis forma pulcherrima. Imaginare amphitheatrum aliquod immensum, et quale sola rerum natura possit effingere. Lata et diffusa planities montibus cingitur, montes summa sui parte procera nemora et antiqua habent. Frequens ibi et uaria uenatio. Inde caeduae siluae cum ipso monte descendunt. Has inter pingues terrenique colles - neque enim facile usquam saxum etiam si quaeratur occurrit - planissimis campis fertilitate non cedunt, opimamque messem serius tantum, sed non minus percoquunt. Sub his per latus omne uineae porriguntur, unamque faciem longe lateque contexunt; quarum a fine imoque quasi margine arbusta nascuntur. Prata inde campique, campi quos non nisi, ingentes boues et fortissima aratra perfringunt: tantis glaebis tenacissimum solum cum primum prosecatur assurgit, ut nono demum sulco perdometur. Prata florida et gemmea trifolium aliasque herbas teneras semper et molles et quasi nouas alunt. Cuncta enim perennibus riuis nutriuntur; sed ubi aquae plurimum, palus nulla, quia deuexa terra, quidquid liquoris accepit nec absorbuit, effundit in Tiberim. Medios ille agros secat nauium patiens omnesque fruges deuehit in urbem, hieme dumtaxat et uere; aestate summittitur immensique fluminis nomen arenti alueo deserit, autumno resumit. Magnam capies uoluptatem, si hunc regionis situm ex monte prospexeris. Neque enim terras tibi sed formam aliquam ad eximiam pulchritudinem pictam uideberis cernere: ea uarietate, ea descriptione, quocumque inciderint oculi, reficientur. Villa in colle imo sita prospicit quasi ex summo. 
La beauté du paysage naturel - comparé d'ailleurs à un tableau - et le plaisir qu'il donne à la vue sont explicitement soulignés dans ce passage. Le goût du paysage apparaît aussi dans la sensualité des descriptions : le sens prédominant est celui de la vue, qui met en exergue l'éclat de la lumière et de la mer ainsi que le contraste entre ombre et lumière ${ }^{29}$, mais le paysage est aussi sonore, car les bruits d'eau, notamment ceux de la mer, sont bien présents et ce paysage devient aussi olfactif, de manière ponctuelle, avec la mention du parfum des violettes sur une terrasse de la villa des Laurentes ${ }^{30}$. Les descriptions des sols font appel au sens tactile ${ }^{31}$.

L'importance accordée aux paysages réels fait écho à celle des paysages peints dans les décors des villas à otium, qui est de plus en plus grande dans la peinture dite du IV e style, qui se développe à partir du règne de Claude ${ }^{32}$. Les principales composantes, chez Pline, de ce que l'on pourrait qualifier de "paysage à otium », constituent aussi les sujets ou les motifs des tableaux paysagers : la mer et les jardins, naturellement ${ }^{33}$, mais aussi la montagne et la plaine ${ }^{34}$, les éléments architecturaux et les villas, dont le plaisir qu'ils procurent à la vue est explicitement souligné dans les lettres de Pline ${ }^{35}$. On peut

29 Sur l'importance accordée à l'exposition des différentes pièces, Colum. 1.6.1-3.

3o Sur les bruits de l'eau: Plin. Epist. 2.17.22, sur le grondement de la mer et l'agitation des tempêtes ; 5.6.23: «une pièce d'eau placée en-dessous des fenêtres et en contrebas, qui fait le plaisir des oreilles et des yeux». Sur l'exploitation de l'eau pour construire une « architecture des sens », et notamment sur l'omniprésence des bruits d'eau, Zarmakoupi 2014, op. cit. (n. 10), 229-235. Parfum des violettes : Epist. 2.17.17.

31 Le passage cité de la description du pays autour de la villa de Toscane mentionne les mottes de terre et le sol compact, les herbes tendres et souples : Plin. Epist. 5.6.10-11; voir aussi, dans la description du paysage autour de la villa des Laurentes, la mention du sol doux et souple sous la tonnelle de vigne : Plin. Epist. 2.17.15.

32 Sur l'évolution de la place et de l'importance accordée aux paysages peints dans les décors des villas, voir Croisille 2010, op. cit. (n. 4), 35-50 ; Zarmakoupi 2014, op. cit. (n. 10), 123, qui renvoie à W.J.T. Peters, <Die Landschaft in der Wandmalerei Kampaniens >, dans G. Cerulli Irelli, M. Aoyagi et S. de Caro (éds.), Pompejanische Wandmalerei (Stuttgart et Zürich 1990), 249-262, part. 261-262. Au sein d'une abondante littérature sur les tableaux de paysage, on peut renvoyer, outre à l'étude classique de W.J. Peters (Landscape in Romano-Campanian Mural Painting [Assen 1963]), à celles d'A. Rouveret, notamment, pour une réflexion sur la notion de paysage : Rouveret 2004, op. cit. (n. 4), 341-343 sur la description par Pline du paysage toscan.

33 P. Zanker, Roman Art (Los Angeles 2010), 12. Cf. Plin. Epist. 2.17.15, description d'un jardin intérieur : «C'est de cette vue, qui n'est pas moins plaisante que celle de la mer, que jouit la salle à manger à l'écart de la mer ».

34 Voir le passage cité plus haut (Plin. Epist. 5.6.7-14). La montagne est surtout présente sous forme de paysages rocheux dans les «paysages mythologiques » : cf. Croisille 2010, op. cit. (n. 4), 51-55.

35 Plin. Epist. 2.17.12: « qui domine une vaste étendue de mer, une grande longueur de rivage et de charmantes villas »; $§ 27:$ «Le rivage s'orne en une délicieuse variété d'une suite tantôt ininterrompue tantôt discontinue de constructions qui offrent l'aspect d'une 


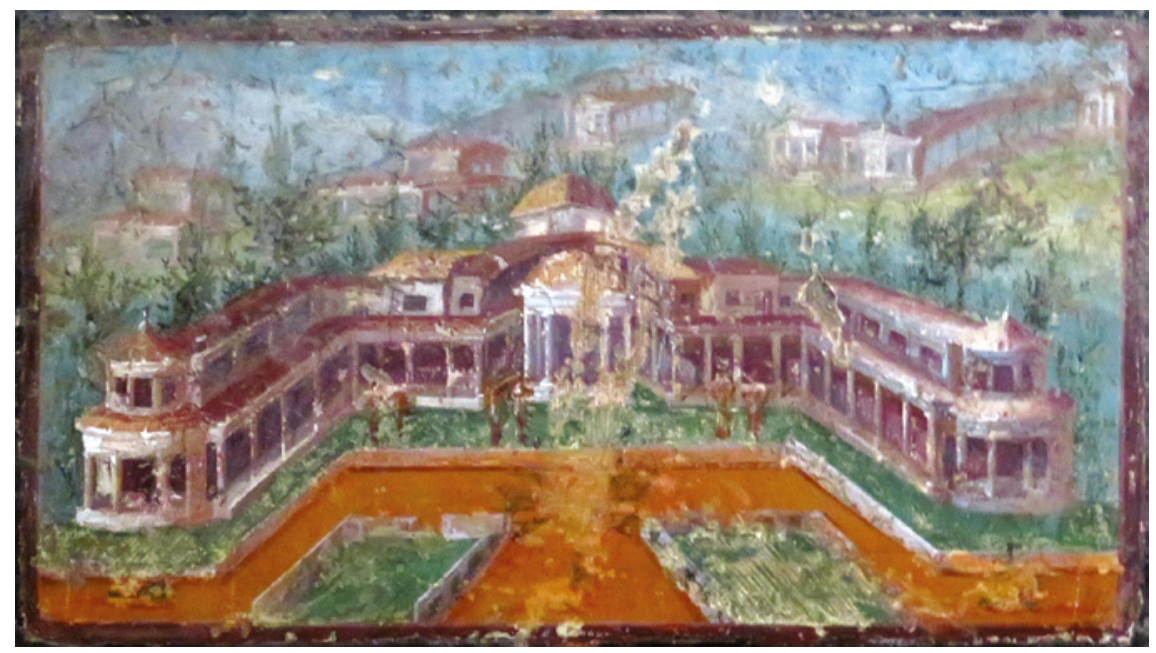

FIGURE 16.1 Pompéi, maison de M. Lucretius Fronto v 4a, tablinum, Villenlandschaft («paysage à villa »)

PHOTOGRAPHIE @ STÉPHANIE WYLER

faire le parallèle entre ce goût pour les paysages comportant des villas et les tableaux de villas du tablinum de la maison de L. Lucretius Fronto, ou bien les panoramas de la maison de la Petite Fontaine à Pompéi ${ }^{36}$. Ces tableaux précèdent de peu dans le temps les descriptions de paysages faites par Pline et par Stace dans les Silves. Le tablinum de Fronto contient la représentation d'une intéressante villa, dont l'architecture, caractérisée par un vaste portique à deux étages, semble ouverte sur le paysage ; le paysage extérieur est marqué par la présence d'autres constructions (notamment des portiques) et de montagnes à l'arrière-plan (fig. 16.1). Le plus grand panorama peint de la maison de la Petite Fontaine embrasse plusieurs constructions : à gauche, une villa avec un grand portique, derrière lequel on aperçoit la végétation d'un jardin ; sur la droite, un petit port semble être représenté (fig. 16.2).

succession de villes »; Plin. Epist. 5.6.30 : «À l'extrémité une chambre, à laquelle la galerie elle-même, autant que les vignes, donnent une vue charmante»; 332 : «L'organisation et la beauté des bâtiments sont, de loin et de très loin, dépassées par celles de l'hippodrome ». Sur les « paysages à villas », ou Villenlandschaften, qui semblent avoir constitué un genre en soi à la fin de la période pompéienne (fin du III ${ }^{\mathrm{e}}$ style-IV ${ }^{\mathrm{e}}$ style), voir Croisille 2010, op. cit. (n. 4), 61 et 112-117. Sur l'importance du critère de visibilité dans le choix du site des villas sur la côte sud du Latium.

36 Voir Croisille 2010, op. cit. (n. 4), 112-113 sur les paysages à villas de la maison de Fronto, et 113-117 sur les panoramas de la maison de la Petite Fontaine (avec des renvois à la bibliographie antérieure). 


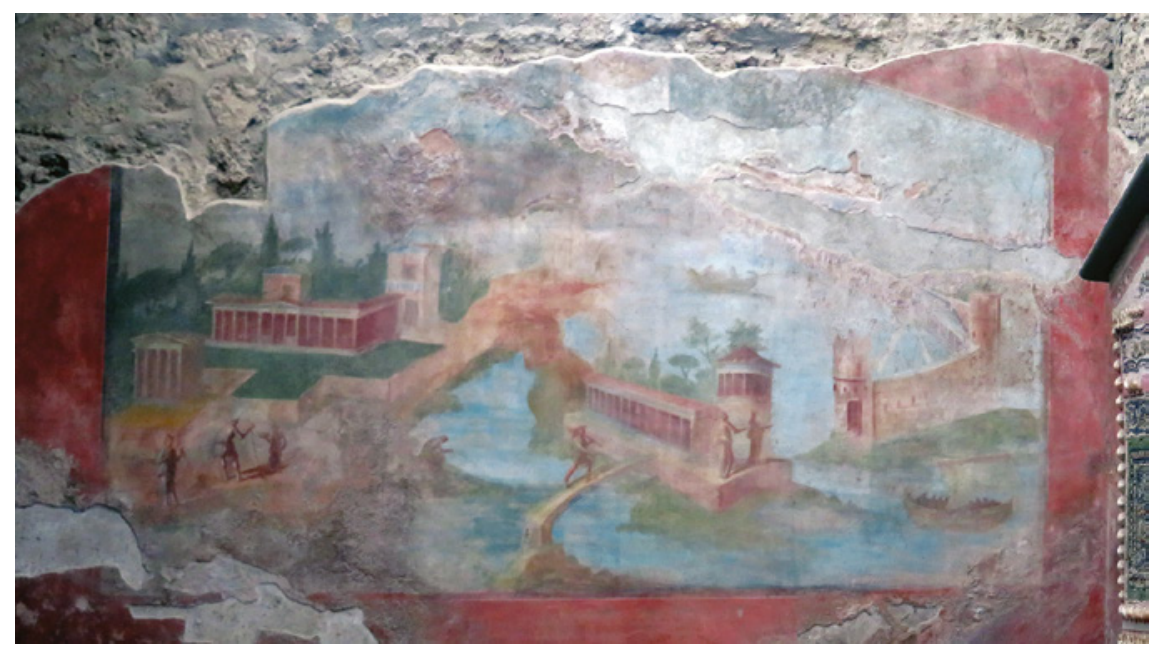

FIGURE 16.2 Pompéi, maison de la Petite Fontaine Vi 8,23, péristyle, panorama PHOTOGRAPHIE C STÉPHANIE WYLER

Le plaisir propre à l'otium n'est pas seulement engendré par la nature aménagée par l'homme, comme on le lit souvent, mais aussi par la nature non aménagée, alternant avec la première ${ }^{37}$. Chez Pline, la nature non aménagée qui apparaît dans le motif de la montagne aux confins du paysage environnant la villa toscane, ou bien par touches dans la description de la villa des Laurentes, est un élément important de contraste avec la nature organisée par l'homme ${ }^{38}$, de même que, dans le «paysage à villa » de la maison de Fronto (fig. 16.1), la végétation vert foncé du jardin qui apparaît derrière le grand portique forme un contraste avec la végétation vert clair et les montagnes grises de l'arrière-plan.

Il est clair qu'on ne saurait reconstituer un unique «paysage à otium», car c'est la variété de l'environnement qui est mise en avant, comme le montre aussi la description que fait Stace, le contemporain de Pline, de la luxueuse villa à Sorrente de son protecteur Pollius Felix, ancien magistrat et patron de

37 Plin. Epist. 5.6.18: «Puis une prairie, qui doit sa beauté à la nature autant que les parties précédentes à l'art». Sur l'importance de la nature aménagée par les hommes, voir par exemple P. Zanker 2010, op. cit. (n. 33), 125. Sur le spectacle de la nature: E. Vallette et S. Wyler (éds.), « Le spectacle de la nature. Regards grecs et romains », Cahiers «Mondes anciens $\gg$ (2017), mis en ligne le 16 mars 2017, consulté le 29 mai 2019. URL : http://jour nals.openedition.org/mondesanciens/1926; DOI : 10.40oo/mondesanciens.1926.

38 N. Purcell, «Alla scoperta di una costa residenziale romana: il litus Laurentinum e l'archeologia dell'otium », dans M.G. Lauro (éd.), Castelporziano III. Campagne di scavo e restauro 1987-1991 (Rome 1998), 11-32, part. 18-19. 


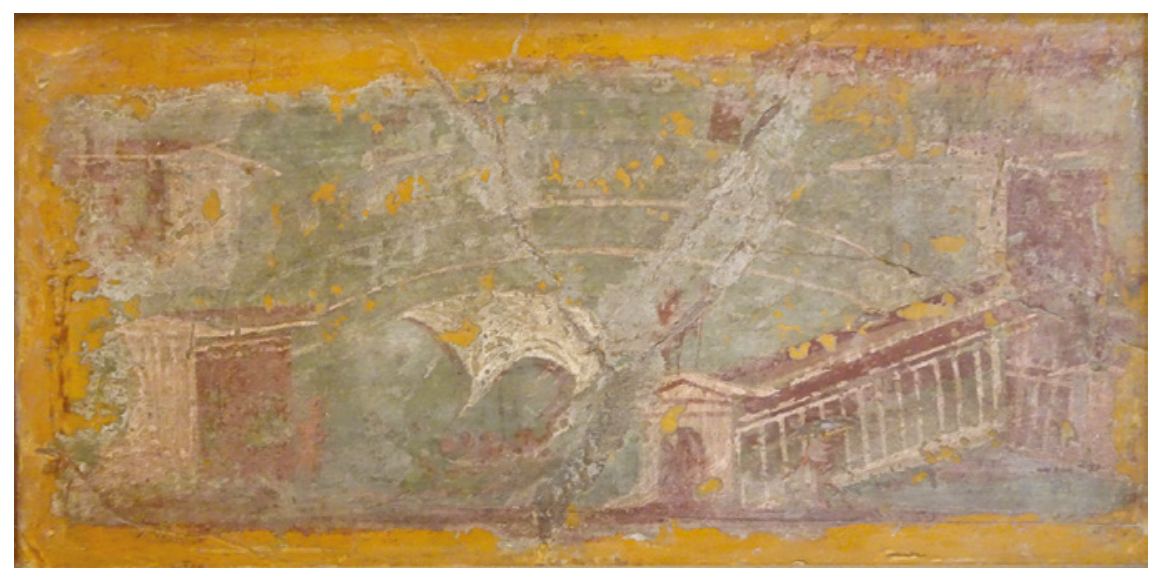

FIgURE 16.3 Pompéi, 45-79 ap. J.-C., paysage portuaire (Naples, MANN, inv. 946o) PHOTOGRAPHIE (C) ANNE GANGLOFF

Pouzzoles et de Naples ${ }^{39}$. Stace met en exergue un autre élément du paysage, le port, qui est aussi un symbole traditionnel de l'otium romain conçu comme un refuge ${ }^{40}$, et qui est également un thème des tableaux peints sur les murs des villas à otium (fig. 16.3). ${ }^{41}$

Il existait un lien évident entre paysages extérieurs, paysages intérieurs peints et paysage mental de l'otium. La notion d'otium qui ressort est celle de

39 Cette variété est soulignée dans Plin. Epist. 2.17.3 et 27 ; 5.6.13; Stat. Silv. 2.2, v. 41-44, 72-74. Sur Pollius Felix, $P I R^{2} \mathrm{P} 55$ o. La variété des points de vue était un critère de l'architecture de la villa des Laurentes, comme on le constate à propos des vues offertes par les pièces du pavillon que Pline a fait lui-même construire, voir par exemple Plin. Epist. 2.17.21 : « À ses pieds on a la mer, derrière soi les villas, en face les bois : autant de paysages différents qu'il distingue et réunit par un nombre équivalent de fenêtres ». Sur cette recherche de points de vue multiples dans la villa maritime, voir Lafon 2001, op. cit. (n. 8), 298-300.

40 Stat. Silv. 2.2, v. 140-141; Cic. Off. 3.2.1 (à propos, justement, de Scipion l'Africain).

41 Sur le port comme élément traditionnel des fresques: Vitr. 7.5.2. Sur le paysage portuaire de Pompéi (Fig. 3), voir I. Bragantini et V. Sampaolo (éds.), La pittura pompeiana (Naples et Milan 2018, $2^{\mathrm{e}}$ éd.), 411. Voir aussi le tableau bien connu de la villa San Marco de Stabies représentant un port qui a été identifié à celui de Pouzzoles ou d'Alexandrie, mais qui est peut-être fictif: Croisille 2010, op. cit. (n. 4), 124-125. Sur les équipements portuaires de certaines villas maritimes: Lafon 2001, op. cit. (n. 8), 320-322. Sur les topia et la description de Stace: B. Bergmann, «Painted perspectives of a villa visit: Landscape as status and metaphor », dans E.K. Gazda et A.E. Haeckl (éds.), Roman Art in the Private Sphere. New Perspectives on the Architecture and Décor of the Domus, Villa, and Insula (Ann Arbor 1991), 49-70, part. 65 sur le port (l'auteur renvoie, n. 40, à C. Bonner, «Desired haven », Harvard Theological Review 34 [1944], 49-67, sur le symbolisme du port). 
délassement de l'esprit, du corps et des sens, mais elle se concilie très bien, chez Pline, avec la conception de l'otium comme étude ${ }^{42}$.

Dans la villa des Laurentes, spécialement dévolue à l'activité littéraire, le sénateur avait fait construire un pavillon isolé par un double mur du bruit de la mer et des esclaves, qui permettait le recueillement propice à l'étude ${ }^{43}$. La lettre de Pline expliquant à Fuscus comment il organisait son otium dans la villa de Toscane souligne l'importance du cadre de l'activité littéraire :

Tu me demandes comment j'organise ma journée en été dans ma propriété de Toscane. Je me réveille quand il me plait, généralement vers la première heure, souvent plus tôt, rarement plus tard. Mes fenêtres restent fermées. Car c'est extraordinaire : bien isolé, grâce au silence et à l'obscurité, de tout ce qui distrait, libre et livré à moi-même, je laisse mes yeux dépendre de ma volonté, non pas ma volonté de mes yeux, qui voient ce que voit mon esprit chaque fois qu'ils n'ont pas autre chose à voir. Je réfléchis à l'ouvrage que j'ai en train, j'y réfléchis jusque dans sa forme, comme si je l'écrivais ou le corrigeais, rédigeant tantôt moins, tantôt plus, selon que le texte a été difficile ou facile à élaborer et à retenir. J'appelle un secrétaire et, après avoir laissé entrer la lumière du jour, je dicte ce que j'ai composé ; il se retire, est de nouveau rappelé, de nouveau renvoyé. À la quatrième ou cinquième heure (car mon emploi du temps n'est pas précis et calculé), selon ce que suggère le ciel, je me rends sur la terrasse ou dans la galerie, je travaille à la suite de mon texte et dicte. Je monte en voiture. Là encore, mon occupation est la même qu'à la promenade ou sur mon lit ; mon attention se maintient, renouvelée par le simple changement. Je dors de nouveau un peu, puis je me promène. Ensuite, je lis un discours grec ou latin distinctement et avec énergie, moins pour ma voix que pour ma gorge ; mais, du même coup, elle aussi s'en trouve fortifiée. Je me promène une nouvelle fois, je me fais frictionner, je fais de l'exercice, je prends un bain. Pendant mon dîner, si je suis avec ma femme ou quelques amis, on lit un ouvrage. Après le dîner vient un comédien ou un joueur de lyre. Ensuite, je me promène avec mes gens, au nombre desquels il en est d'instruits. C'est ainsi que la soirée se prolonge en

Sur la villa des Laurentes consacrée à l'activité littéraire : Plin. Epist. 1.9.4-6 ; 1.22.11 ; 4.6 ; 9.36 ; S.E. Hoffer, The Anxieties of Pliny the Younger (New York 1999), 29-44: «Villas: Factories of literature » (à propos d'Epist. 1.3); P. Grimal, Les jardins romains (Paris 1984, $3^{\mathrm{e}}$ éd.), 423-425, a mis en évidence le lien entre les jardins et l'otium consacré à l'étude ; K.T. von Stackelberg, The Roman Garden: Space, Sense, and Society (London et New York 2009), 125-134, sur les jardins de la villa toscane de Pline. 
conversations variées et que, quand bien même est-il fort long, le jour s'achève vite. Il y a parfois dans cette régularité quelques changements. Car, si je suis resté longtemps au lit ou à la promenade, c'est seulement après ma sieste et ma lecture que je me déplace, non en voiture, mais, ce qui prend moins de temps grâce à une plus grande rapidité, à cheval. Des amis surviennent-ils des villes voisines? Ils me prennent une partie de ma journée et quelquefois, si je suis fatigué, viennent à mon secours en m'interrompant opportunément. Je chasse de temps en temps, mais sans oublier mes tablettes, pour que, même si je ne prends rien, je ne revienne pas sans rien. Je donne aussi du temps à mes fermiers, pas assez à leurs yeux; et leurs doléances de paysans me font apprécier davantage nos travaux littéraires et les occupations de citadins qui sont les nôtres. Au revoir 44 .

Pline commence ainsi, dès l'aube, à composer des ouvrages dans la solitude et l'obscurité d'une chambre fermée : curieuse habitude, que permet de comprendre le témoignage de son maître Quintilien sur la conception traditionnelle, grecque - le modèle en est Démosthène -, du travail littéraire.

44 Plin. Epist. 9.36, Pline à Fuscus: Quaeris quemadmodum in Tuscis diem aestate disponam. Euigilo cum libuit, plerumque circa horam primam, saepe ante, tardius raro. Clausae fenestrae manent; mire enim silentio et tenebris ab iis quae auocant abductus et liber et mihi relictus, non oculos animo sed animum oculis sequor, qui eadem quae mens uident, quotiens non uident alia. Cogito si quid in manibus, cogito ad uerbum scribenti emendantique similis, nunc pauciora nunc plura, ut uel difficile uel facile componi teneriue potuerunt. Notarium uoco et die admisso quae formaueram dicto; abit rursusque reuocatur rursusque dimittitur. Vbi hora quarta uel quinta - neque enim certum dimensumque tempus - ut dies suasit, in xystum me uel cryptoporticum confero, reliqua meditor et dicto. Vehiculum ascendo. Ibi quoque idem quod ambulans aut iacens; durat intentio mutatione ipsa refecta. Paulum redormio, dein ambulo, mox orationem Graecam Latinamue clare et intente non tam uocis causa quam stomachi lego; pariter tamen et illa firmatur. Iterum ambulo ungor exerceor lauor. Cenanti mihi, si cum uxore uel paucis, liber legitur; post cenam comoedia aut lyristes; mox cum meis ambulo, quorum in numero sunt eruditi. Ita uariis sermonibus uespera extenditur, et quamquam longissimus dies bene conditur. Non numquam ex hoc ordine aliqua mutantur; nam, si diu iacui uel ambulaui, post somnum demum lectionemque non uehiculo sed, quod breuius quia uelocius, equo gestor. Interueniunt amici ex proximis oppidis, partemque diei ad se trahunt interdumque lasso mihi opportuna interpellatione subueniunt. Venor aliquando, sed non sine pugillaribus, ut quamuis nihil ceperim non nihil referam. Datur et colonis, ut uidetur ipsis, non satis temporis, quorum mihi agrestes querelae litteras nostras et haec urbana opera commendant. Vale. Voir aussi Epist. 9.40, à Fuscus, où Pline affirme qu'il règle son otium à peu près de la même façon l'hiver, quand il séjourne dans sa villa des Laurentes. 
L'absence de témoins [...] et le plus profond silence sont, personne n'en doute, les meilleures conditions pour écrire. Ce n'est pas à dire qu'il faille du coup écouter ceux pour qui les bois et les forêts conviennent le mieux à cette fin, sous prétexte que le ciel libre et l'agrément du paysage élèvent l'âme et enrichissent l'inspiration. Pour moi, du moins, cette solitude est agréable plus que stimulante pour l'étude. En fait, ces spectacles, précisément parce qu'ils charment, détournent nécessairement de s'appliquer au travail que l'on a en vue. En effet, l'esprit ne peut s'appliquer consciencieusement tout entier à beaucoup d'objets à la fois, et, où qu'il se tourne, il cesse de fixer le but qu'il s'était proposé. Aussi, l'agrément des forêts et les eaux qui coulent et le souffle des brises dans les branches d'arbres et le chant des oiseaux, et simplement la liberté même de promener largement nos regards autour de nous, tout nous attire, en sorte que, pour moi, ce plaisir me semble plutôt détendre l'esprit que le tendre. Démosthène était plus sage, lui, qui s'enfermait dans un lieu d'où il ne pût rien entendre ni regarder, de peur que ses yeux ne le contraignent à penser à autre chose. Donc, si nous veillons, que ce soit de préférence le silence de la nuit, et une chambre bien close, avec une lampe solitaire qui nous fassent comme un abri ${ }^{45}$.

On retrouve dans cette conception traditionnelle de létude la notion de l'otium intellectuel défendue par Sénèque, en relation avec le cadre clos et sombre qu'il appréciait dans la villa de Scipion l'Africain. Quintilien, également favorable à cette conception de létude, présente aussi une position opposée, dont les partisans défendaient l'idée que le paysage peut inspirer l'activité littéraire, stimulée par le plaisir d'un environnement plein de charme ${ }^{46}$.

45 Quint. Inst. 10.3.22-25: Secretum [...] et quam altissimum silentium scribentibus maxime conuenire nemo dubitauerit. Non tamen protinus audiendi qui credunt aptissima in hoc nemora siluasque, quod illa caeli libertas locorumque amoenitas sublimem animum et beatiorem spiritum parent. Mihi certe iucundus hic magis quam studiorum hortator uidetur esse secessus. Namque illa quae ipsa delectant necesse est auocent ab intentione operis destinati. Neque enim se bona fide in multa simul intendere animus totum potest, et quocumque respexit desinit intueri quod propositum erat. Quare siluarum amoenitas et praeterlabentia flumina et inspirantes ramis arborum aurae uolucrumque cantus et ipsa late circumspiciendi libertas ad se trahunt, ut mihi remittere potius uoluptas ista uideatur cogitationem quam intendere. Demosthenes melius, qui se in locum ex quo nulla exaudiri uox et ex quo nihil prospici posset recondebat, ne aliud agere mentem cogerent oculi. Ideoque lucubrantes silentium noctis et clusum cubiculum et lumen unum uelut + rectos + maxime teneat. (trad. par J. Cousin).

46 Sur le topos de la solitude des bois comme lieu de la création poétique, Hor. epist. 1.4.1-5, peut-être à propos du poète élégiaque Tibulle. Il s'oppose en tout cas lui-même, dans son 
La méthode de Pline constitue une sorte de mixte, par adaptation aux activités aristocratiques de l'otium qu'étaient les promenades à pied, en litière ou bien à cheval, au sein de la villa ou bien aux alentours. Une deuxième partie de son travail de composition prend place en effet, selon les conditions météorologiques, sur la terrasse ou bien dans la galerie qui impliquaient des vues variées sur le paysage. Même à la chasse, Pline emportait ses tablettes, ce qui signifie qu'il travaillait aussi dans la nature et les bois ${ }^{47}$. Cette attention portée à l'exercice et à la variété des activités correspond à des règles bien établies de la diététique médicale ${ }^{48}$. Mais la variété, le changement, sont également mis en avant comme ressorts de la méthode de travail de Pline: le changement le stimule intellectuellement et lui permet de maintenir son attention. Dans une autre lettre consacrée à la villa des Laurentes, le rôle inspirateur du paysage maritime est directement évoqué : «Ô mer, ô rivage, ô sanctuaire des Muses, authentique et solitaire, qu'elles sont nombreuses, vos découvertes, que de pensées vous m'inspirez ${ }^{49}$.»

D'un Sénèque exprimant son malaise vis-à-vis des villas à otium, lieux où le luxe et les paysages entravent l'étude du sage, à un Pline le Jeune dont les villas des Laurentes et de Toscane constituent non seulement le cadre, mais aussi le moteur et même le sujet de l'activité intellectuelle et littéraire. En explorant les rapports de l'élite romaine aux paysages naturels et peints dans le cadre des

otium sabin créatif, à ce poète qui erre, silencieux, dans les bois salubres : voir Leach 1988, op. cit. (n. 24), 238, qui fait l'hypothèse d'une allusion à l'usage hellénistique et républicain de la nature comme miroir subjectif de l'émotion. Voir aussi Tac. dial. 12.

47 Voir déjà Cic. leg. 2.1, sur son plaisir de réfléchir, écrire et lire dans l'île du Fibrène sur son domaine d'Arpinum.

48 Voir Cels. 1.1.1, sur l'homme en bonne santé : Hunc oportet uarium habere uitae genus: modo ruri esse, modo in urbe, saepiusque in agro ; nauigare, uenari, quiescere interdum, sed frequentius se exercere ; siquidem ignauia corpus hebetat, labor firmat, illa maturam senectutem, hic longam adulescentiam reddit, «Ce qu'il lui faut, c'est de la variété dans sa façon de vivre : être tantôt à la campagne, tantôt en ville, et plus souvent aux champs ; naviguer, chasser, prendre parfois du repos, mais plus fréquemment de l'exercice; car l'inaction alanguit le corps, l'effort l'affermit, la première hâte la vieillesse, l'autre prolonge la jeunesse » (trad. par G. Serbat). Le danger du travail intellectuel qui n'est pas favorable à la bonne santé du corps est souligné par Cels. praef. 6 ; 1.2.1. Voir D. C. 71.36.2, imputant l'affaiblissement du corps de Marc Aurèle à l'étude et à l'ascèse. Marc Aurèle, dans une lettre à Fronton (Fronto 5.63, Van den Hout p. 83, 4), affirme dicter en se promenant, comme l'exigeait le soin de son corps.

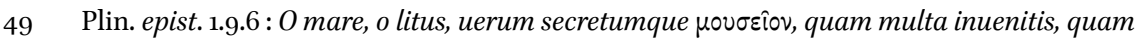
multa dictatis! Sur les liens entre otium, studia et lieux, voir aussi N. Méthy, Les lettres de Pline: une représentation de l'homme (Paris 2007), 374-378. André 1966, op. cit. (n. 1), 515-516, a souligné l'existence, chez Virgile, de paysages dans lesquels des éléments de la nature, en particulier la mer et le ciel, sont associés aux notions de calme, de repos et de sérénité. 
villas à otium, on a donc vu l'évolution des fonctions de ces villas au $\mathrm{I}^{\mathrm{er}}$ siècle ap. J.-C. Ces changements font intervenir des facteurs aussi bien culturels que politiques: processus d'appropriation du paysage, conceptions de l'otium et rapports au pouvoir impérial.

Ainsi, l'évolution de l'architecture et du décor de la villa à otium, celle de l'appréhension du paysage et celle de la notion d'otium sont allées de pair. Une nouvelle sensibilité à l'environnement s'exprime dans le goût sensuel pour le paysage, qui est intégré à la notion d'otium et donne naissance à une nouvelle façon d'envisager l'étude. Le paysage n'est plus seulement conçu comme reflet des sentiments et des émotions, comme c'était le cas à l'époque hellénistique et républicaine (on peut l'observer d'ailleurs dans la correspondance de Cicéron ${ }^{50}$ ). Il est aussi revendiqué, de façon explicite, comme le cadre et le ressort psychologique de l'activité intellectuelle. D'où une nouvelle manière de concevoir cette activité, dans laquelle l'étude est stimulée par le mouvement et par la variété de l'environnement, et où des éléments naturels deviennent une source d'inspiration revendiquée.

Alors qu'elles étaient auparavant surtout le cadre des conversations érudites, les villas à otium sont donc devenues, à la fin du $\mathrm{I}^{\mathrm{er}}$ siècle ap. J.-C., le cadre des études personnelles et de la création littéraire, comme on le voit aussi chez Stace (Silv., 2.2) où le paysage maritime de la villa de Pollius Felix est une composante à part entière du cadre des études astronomiques, philosophiques et poétiques de son propriétaire ${ }^{51}$. À l'issue d'une longue construction culturelle

$5^{\circ} \quad$ Leach 1988, op. cit. (n. 24), 138. Sur les paysages de Cicéron qui traduisent ses préoccupations politiques, J.-P. De Giorgio, « Sous l'épopée, la plage », Cahiers «Mondes anciens » 9 (2017), mis en ligne le 07 mars 2017, consulté le 29 mai 2019. URL : http://journals.open edition.org/mondesanciens/1898; DOI : 10.400o/mondesanciens.1898.

51 Voir aussi Plin. epist. 1.3. Stace décrit le paysage maritime de la villa comme un paysage à la fois naturel et mythologique, évoquant celui des tableaux peints qui relèvent de la catégorie dite des «paysages mythologiques », où le paysage naturel est le décor évocateur et, plus précisément, le point d'ancrage des épisodes mythiques : voir par exemple les deux tableaux de la villa Boscotrecase représentant, l'un, le mythe de Polyphème et Galatée, l'autre, celui de Persée et Andromède, datés entre 15 av. J.-C. et 10 ap. J.-C. (Croisille 2010, op. cit. [n. 4], 93-98). La fin du poème de Stace (v. 112-121) fait apparaître le lien entre l'activité poétique de Felix et la dimension mythologique du paysage maritime. Stace ne manque naturellement pas d'évoquer les Sirènes de Sorrente; voir Lafon 2001, op. cit. (n. 8), 199-203, sur l'enjeu socio-culturel des références mythiques à propos des villas maritimes, dont les propriétaires souhaitaient se rattacher aux recherches hellénistiques sur la géographie des mythes. Voir aussi A. Rouveret, « Les paysages de Philostrate », dans M. Costantini, E. Graziani et S. Rolet (éds.), Le défi de l'art. Philostrate, Callistrate et l'image sophistique (Rennes 2006), 63-76, part. 66-67 sur le paysage et la mémoire; A. Rouveret, « Retour à Ithaque : peinture du paysage et de l'intimité domestique à Rome du dernier siècle de la République au début de l'Empire », CRAI 2013.1, 289-312. Voir également 
du paysage ${ }^{52}$, celui-ci apparait désormais comme un élément à part entière et un élément dynamique - de la culture et de l'otium romains.

Dans ce processus d'appropriation du paysage par l'élite romaine, le $\mathrm{I}^{\mathrm{er}}$ siècle semble représenter une étape importante, comme le montre le fait que les villas à otium et le luxe ne semblent plus contestés à partir des Flaviens ${ }^{53}$. L'analyse des réactions de Sénèque et de Pline le Jeune permet de comprendre les différents éléments qui ont interagi dans ce processus: le développement du goût pour le paysage intégré dans l'architecture des villas à otium, la «normalisation» des rapports entre l'élite intellectuelle sénatoriale et le prince, la diffusion de nouveaux modèles de l'otium, pour lesquels l'otium des villas impériales a sans aucun doute fourni les principaux critères, et de nouvelles conception et pratique de l'étude au sein-même des villas. Ces réactions prennent leur sens dans le contexte de la perte générale de pouvoir politique qu'a vécue l'ordre sénatorial au début du Principat. La réalisation de l'otium intellectuel au sein des villas italiennes, dans le cadre d'un paysage familier à l'aristocratie romaine, devient alors un éventuel exutoire pour les sénateurs. Sous Néron et les Flaviens, cette perte du pouvoir politique a engendré des réactions conflictuelles, dont les plus fameuses sont les actes d'opposition des « sénateurs stoïciens ». Sénèque ne faisait pas partie de ce groupe, mais l'otium des villas de luxe lui était d'une certaine façon imposé par Néron : il devenait de ce fait (outre des raisons proprement philosophiques) inacceptable, à partir du moment où le sénateur s'était éloigné du pouvoir. La période flavienne a implanté de manière décisive le Principat dans la culture politique romaine. Pline, qui a fait les débuts prometteurs de sa carrière politique sous Domitien, et qui décrit ses villas au début de lépoque antonine, dans une période de compromis politique, n'a plus aucune réticence à exploiter et louer les possibilités d'activités que la villa à otium offrait aux membres de l'ordre sénatorial.

dans ce volume la contribution d'A. Walker, The landscape and nature of the Cyclops in Campanian wall-painting.

$5^{2}$ Sur la construction culturelle et économique du paysage du littoral laurentin, Purcell 1998, op. cit. (n. 38).

53 Edwards 1993, op. cit. (n. 11), 142 et 163; Lafon 2001, op. cit. (n. 8), 285: « Les obstacles intellectuels à la possession de villas maritimes ont totalement disparu dans la deuxième moitié du I $^{\text {er }}$ siècle ap. J.-C. Ce retournement complet est inséparable d'une nouvelle approche de l'otium, elle-même liée au nouveau système politique ». 